\title{
A Novel Balloon Assisted Two-Stents Telescoping Technique for Repositioning an Embolized Stent in the Pulmonary Conduit
}

\author{
Daisuke Kobayashi, ${ }^{*}$ MD, Srinath T. Gowda, MD, and Thomas J. Forbes, MD, FSCAI
}

\begin{abstract}
A 9-year-old male, with history of pulmonary atresia and ventricular septal defect, status post complete repair with a $16 \mathrm{~mm}$ pulmonary homograft in the right ventricular outflow tract (RVOT) underwent 3110 Palmaz stent placement for conduit stenosis. Following deployment the stent embolized proximally into the right ventricle (RV). We undertook the choice of repositioning the embolized stent into the conduit with a transcatheter approach. Using a second venous access, the embolized stent was carefully maneuvered into the proximal part of conduit with an inflated Tyshak balloon catheter. A second Palmaz 4010 stent was deployed in the distal conduit telescoping through the embolized stent. The Tyshak balloon catheter was kept inflated in the RV to stabilize the embolized stent in the proximal conduit until it was successfully latched up against the conduit with the deployment of the overlapping second stent. One year later, he underwent Melody valve implantation in the pre-stented conduit relieving conduit insufficiency. This novel balloon assisted two-stents telescoping technique is a feasible transcatheter option to secure an embolized stent from the RV to the RVOT. ๑ 2014 Wiley Periodicals, Inc.
\end{abstract}

Key words: stent embolization; pediatric intervention; balloon assist; two-stents telescoping

\section{INTRODUCTION}

Transcatheter stent placement has evolved as an important therapeutic option in the management of children with congenital heart disease [1]. Although stent placement in the right ventricular outflow tract (RVOT) is a routine procedure and is mostly performed safely, inadvertent embolization of an expanded stent into the right ventricle $(\mathrm{RV})$ occurs infrequently. The management of an embolized stent is particularly challenging as repositioning the expanded stent is often difficult and potentially incurs injury to the cardiac apparatus. Previously reported transcatheter techniques involve withdrawing an embolized stent from the RV to right atrium and inferior vena cava [2-5]. However, there is a risk of causing injury to the tricuspid valve leaflets and may require open heart surgery to exteriorize the stent.

We report an inadvertent embolization of a 3110 Palmaz stent from the pulmonary artery conduit into the $\mathrm{RV}$ in a young child. The embolized stent was successfully repositioned in the pulmonary conduit using a novel balloon assisted two-stents telescoping technique.

\section{CASE REPORT}

A 9-year-old male weighing $37 \mathrm{~kg}$ was born with pulmonary atresia, ventricular septal defect (VSD) and major aorto-pulmonary collateral arteries. He underwent initial surgery involving unifocalization of pulmonary arteries, fenestrated VSD closure, and placement of pulmonary conduit at 5 months of age. He underwent replacement of the pulmonary conduit with a $16 \mathrm{~mm}$ pulmonary homograft at 9 months of age for a large pseudoaneurysm in the RVOT. Subsequently, for right pulmonary artery (RPA) stenosis, a $25 \mathrm{~mm}$

Division of Pediatric Cardiology, Children's Hospital of Michigan, Carman and Ann Adams Department of Pediatrics, Wayne State University School of Medicine, Detroit, Michigan

Additional supporting information may be found in the online version of this article.

Conflict of interest: Nothing to report.

*Correspondence to: Daisuke Kobayashi, Division of Pediatric Cardiology, Children's Hospital of Michigan, Carman and Ann Adams Department of Pediatrics, Wayne State University School of Medicine 3901 Beaubien Blvd, Detroit, MI 48201-2119. E-mail: dkobayas@dmc.org

Received 30 October 2013; Revision accepted 3 January 2014

DOI: $10.1002 / \mathrm{ccd} .25383$

Published online 9 January 2014 in Wiley Online Library (wileyonlinelibrary.com) 


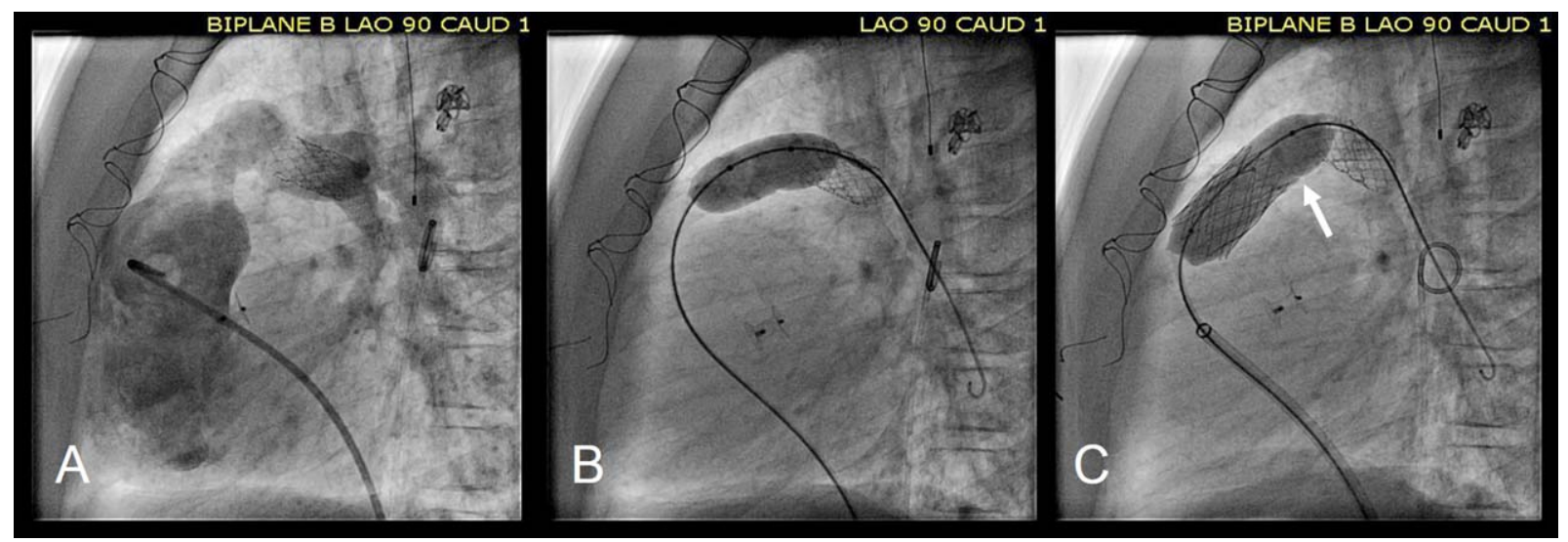

Fig. 1. Straight lateral projection at the initial stent deployment. A: Angiography in the RV shows stenotic pulmonary conduit with the narrowest site measuring $12.5 \mathrm{~mm}$. Note the previously implanted $25 \mathrm{~mm}$ Genesis XD stent in the proximal RPA. B: Balloon angioplasty shows the waist at the mid conduit stenosis. C: The stent migrates proximally and slips from the stenotic lesion (arrow) during the final inflation. [Color figure can be viewed in the online issue, which is available at wileyonlinelibrary.com.]

Genesis XD stent was placed in the proximal RPA at 8 years of age.

At 9 years of age, he became symptomatic with worsening fatigue. Echocardiography showed free conduit insufficiency with significantly increased right ventricular systolic pressure $(55 \mathrm{~mm} \mathrm{Hg})$ and moderate conduit stenosis (peak gradient of $44 \mathrm{~mm} \mathrm{Hg}$ ). Hemodynamic study revealed elevated RV systolic pressure at $84 \mathrm{~mm} \mathrm{Hg}(68 \%$ systemic) and a gradient of $31 \mathrm{~mm}$ $\mathrm{Hg}$ across the conduit. Angiography demonstrated stenosis in the distal pulmonary conduit measuring $12.5 \mathrm{~mm}$ at the narrowest site (Fig. 1A). Initially, angioplasty was performed to relieve the conduit stenosis using a $15 \mathrm{~mm} \times 2 \mathrm{~cm}$ Maxi $\mathrm{D}$ balloon angioplasty catheter inflated to a maximum of 6.3 atmospheres. The residual waist at full inflation measured $13.1 \mathrm{~mm}$ (Fig. 1B). In the absence of hemodynamic and angiographic improvement, we choose to treat the stenotic conduit with a stent placement. A $0.035^{\prime \prime}$ Rosen wire was positioned in the distal RPA. Using a 12-Fr Flexor sheath, a Palmaz 3110 stent was mounted on a $16 \mathrm{~mm} \times 4 \mathrm{~cm}$ Z-Med II balloon catheter and positioned across the conduit. The stent was deployed after confirming optimal positioning with serial hand injections. The stent was noted to have migrated slightly proximally during the final inflation (Fig. 1C) and subsequently embolized into the RV with balloon deflation (Fig. 2A and Supplemental Video 1).

We initially attempted to reposition the embolized stent in the inferior vena cava. After a few unsuccessful attempts, we aborted the procedure to avoid potential injury to the tricuspid valve apparatus. The decision was made to attempt and reposition the embolized stent across the conduit before proceeding toward surgical extraction. Although the stent was not in the intended site the primary wire remained in position through the stent in the distal RPA. An attempt to partially inflate the $16 \mathrm{~mm} \times 4 \mathrm{~cm} \mathrm{Z}$-med balloon followed by $15 \mathrm{~mm} \times 2 \mathrm{~cm}$ maxi $\mathrm{D}$ balloons within the stent and advance into the conduit were unsuccessful. Further maneuvers including looping the wire in the right atrium and efforts to advance the balloon stent unit across the RVOT to the proximal conduit were ineffective. We felt that the fully expanded stent could not be advanced to the distal conduit and sustain a stable stent position without additional support. A second venous access was obtained. Using a 7-Fr sheath, a 7-Fr Berman wedge catheter was advanced from the femoral vein into the RV. We successfully advanced the catheter through the lumen of the stent, across the conduit and positioned in the distal RPA. Through this catheter, a second $0.035^{\prime \prime}$ Rosen wire was positioned in the distal RPA. The catheter was exchanged for a $16 \mathrm{~mm} \times 4 \mathrm{~cm}$ Tyshak II balloon catheter. The balloon was inflated in the RV and the balloon was advanced to make contact with the proximal part of the embolized stent in the RV (Fig. 2). The balloon catheter was advanced over the wire and with careful manipulation the embolized stent was pushed out into the proximal conduit. We were unable to further advance the stent into the distal conduit; however we were able to maintain a relatively stable stent position in the RVOT and proximal conduit. To prevent proximal migration of the stent back into the RV the balloon catheter remained inflated in the RV holding the stent in the proximal conduit. We decided to deploy a second stent in the distal conduit and secure the embolized stent. Using a 12-Fr Flexor sheath through the

Catheterization and Cardiovascular Interventions DOI 10.1002/ccd.

Published on behalf of The Society for Cardiovascular Angiography and Interventions (SCAI). 


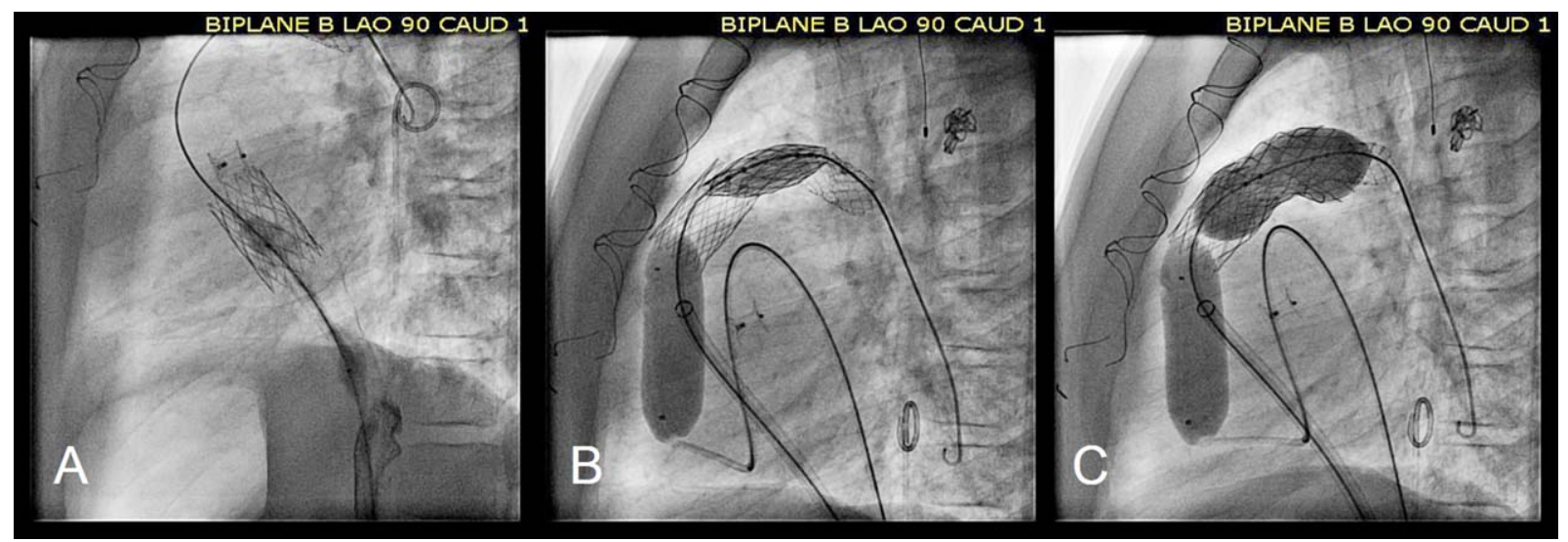

Fig. 2. Straight lateral projection during repositioning the embolized stent. A: The fully expanded stent is embolized into the RV. The wire remains through the lumen of the stent. B, C: While the embolized stent is held in the proximal conduit with an assist of inflated Tyshak II balloon catheter, a second stent is carefully positioned and deployed successfully; it adheres onto the conduit stenosis superiorly and latches on to distal portion of the embolized stent in the RVOT in a stable position. [Color figure can be viewed in the online issue, which is available at wileyonlinelibrary.com.]
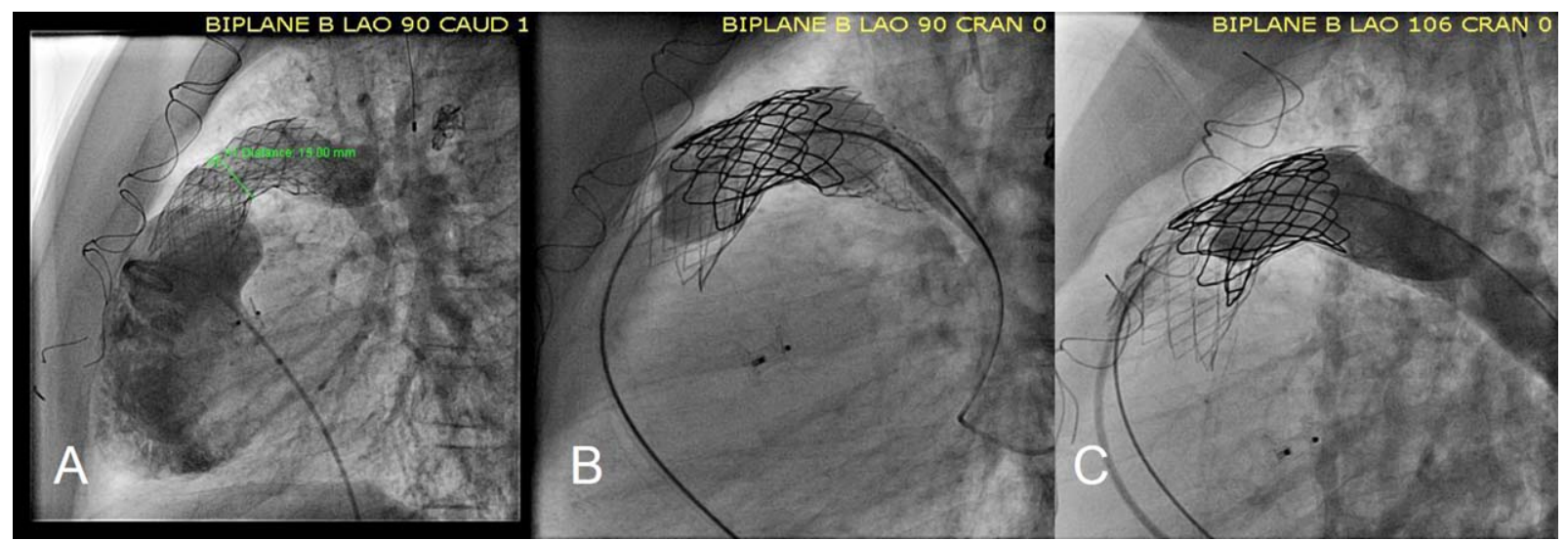

Fig. 3. Straight lateral projection projections after stent repositioning (A) and Melody valve implantation (B and C). A: Two stents telescopes remain in a stable position. The narrowest diameter measures $15 \mathrm{~mm}$. B, C: One year later, Melody valve is successfully implanted in the stented conduit. Note no conduit insufficiency. [Color figure can be viewed in the online issue, which is available at wileyonlinelibrary.com.]

primary wire, a second Palmaz 4010 stent was mounted on an oversized $22 \mathrm{~mm} \times 4 \mathrm{~cm}$ BIB balloon catheter and advanced into the distal conduit telescoping through the embolized stent. The second stent was then carefully inflated to overlap at least $1 / 3 \mathrm{rd}$ of the embolized stent proximally and the remaining stent latched onto the conduit distally. The $16 \mathrm{~mm} \times 4 \mathrm{~cm}$ Tyshak II balloon catheter remained inflated in the RV through the secondary wire securing the embolized stent in the RVOT in a stable position until it was safely tacked up against the conduit by the second stent deployment (Fig. 2B and C and Supplemental Video 2). At the time of stent deployment, transient hypotension was noted that resolved following defla- tion of the balloon. Final angiography demonstrated two overlapping stents secured in a stable position across the conduit (Fig. 3A). A follow-up echocardiogram showed absence of pericardial effusion or any worsening of tricuspid valve regurgitation and the stents were felt to be in a stable position across the pulmonary conduit. One year later, he underwent uneventful Melody valve implantation in the prestented conduit (Fig. 3B and C).

\section{DISCUSSION}

We report a novel balloon assisted two-stents telescoping technique that enabled us to reposition an

Catheterization and Cardiovascular Interventions DOI 10.1002/ccd. Published on behalf of The Society for Cardiovascular Angiography and Interventions (SCAI). 
accidentally embolized expanded stent from the RV to the pulmonary conduit successfully without complications. In contrast to traditional approaches, this technique allowed an embolized stent to be cautiously maneuvered into the RVOT away from the tricuspid valve, thereby reducing the risk of tricuspid valve injury. Our case highlights the complexity in managing an inadvertently embolized stent in the RV. This provides an alternative transcatheter approach in managing an embolized stent in the RV and thus preventing an emergent heart surgery or alternative catheterization approach that can potentially cause cardiac injury.

Careful anatomical assessment and procedural planning is critical for the stent placement. Stent dislodgement, malposition, and embolization are unfavorable complications during stent deployment. In our case, stent migrated proximally following balloon inflation. The overall conduit length, and distance between the conduit stenosis and origin of the stented RPA were relatively short. Accordingly, the distal end of balloon catheter pushed against the relatively smaller caliber proximal RPA (RPA measured $12 \mathrm{~mm}$ and the balloon used for stent deployment was $15 \mathrm{~mm}$ ), resulting in the proximal movement of balloon/stent unit following complete inflation. Recognition of relatively short main pulmonary artery segment and choosing a shorter length balloon catheter may have potentially prevented this complication. A Palmaz 3110 stent can be mounted on $3 \mathrm{~cm}$-length balloon catheter.

We used "balloon assisted two-stents telescoping technique" in which an inflated balloon catheter allowed an embolized stent to be positioned in the RV outflow tract. A second stent was deployed distally to overlap and secure the embolized stent and stabilize the entire unit against the stenotic conduit. Narsupalli et al. reported two-stents telescoping technique for securing an atrially embolized stent in a 22-year old with coronary sinus stenosis after Fontan operation [6]. The catheter and wire were positioned from the left internal jugular vein to left superior vena cava, coronary sinus, and atrium. A Genesis Palmaz PG2910B stent mounted on a $16 \mathrm{~mm}$ Cristal balloon (Balt extrusion, Montmorency, France) was dislodged distally while deploying the stent and embolized into the atrium. A second stent (Genesis Palmaz PG395P) mounted on $18 \mathrm{~mm} \times 4 \mathrm{~cm}$ Cristal balloon was deployed to cover the coronary sinus ostial stenosis with its proximal end as well as to engaging the proximal one-third of the embolized stent with its distal end. We employed a similar technique in repositioning the embolized stent from the RV into the RVOT. We performed an additional maneuver by using an inflated balloon catheter to mobilize and steady the embolized stent in the proximal pulmonary conduit. This balloon assist maneuver allowed us to stabilize the embolized stent while deploying the second stent. The second stent successfully overlapped the embolized stent proximally and anchored against the stenotic conduit distally.

Other therapeutic option is retrieval and repositioning of stent to the inferior vena cava (IVC). Balloon catheter is used to engage an embolized stent and balloon/stent unit is withdrawn to the right atrium and IVC. We have reported successful withdrawal of an embolized stent from the RV across the tricuspid valve into the IVC without causing any injury in a small infant [2]. However, there is a strong possibility to injure the cardiac structures-especially the tricuspid valve-with the free edges of the stents. If an expanded stent is too large, there is an option to crimp stent onto the balloon with a gooseneck snare. Portugaller et al. reported this technique in a 58-year-old man with right common iliac artery stenosis, in whom a $7 \mathrm{~mm} \times 24 \mathrm{~mm}$ Palmaz Genesis stent was crimped on a partially inflated $8 \mathrm{~mm} \times 60 \mathrm{~mm}$ balloon catheter with a $2.5 \mathrm{~cm}$ Amplatz gooseneck snare [3]. This balloon/stent unit was pulled back into the right common iliac artery, resulting in the successful stent implantation at the target lesion. Compared to these options, a major advantage with our technique is to avoid injury to the tricuspid valve by repositioning the embolized stent in the RVOT which was the primary purpose of this catheterization and an intended target. By successfully securing the stents a stable pre-stent platform was established in the RVOT, albeit with multiple stents for an eventual deployment of the Melody valve. However, this technique cannot be used or attempted for an embolized stent in the RV in a patient with a competent or native pulmonary valve. Although we did not encounter any major complications, we need to be aware of propensity for ventricular arrhythmias, tricuspid valve injury, and hypotension due to compression of tricuspid valve from two sheaths and cardiac arrest from low cardiac output with a balloon in the RV and a second balloon being inflated for deployment of the second stent. If multiple attempts to stabilize an embolized stent are unsuccessful, emergent surgery to retrieve the stent should be the next prudent option.

\section{CONCLUSION}

This novel balloon assisted two-stents telescoping technique is a feasible alternative in rescuing a challenging stent embolization. This would provide an additional option for an interventional cardiologist in managing a difficult inadvertent stent embolization into the RV, prior to consideration of surgical extraction. 


\section{ACKNOWLEDGMENT}

Authors thank Dr. Daniel R. Turner who provided intellectual insight in preparation of this manuscript.

\section{REFERENCES}

1. Feltes TF, Bacha E, Beekman RH III, Cheatham JP, Feinstein JA, Gomes AS, Hijazi ZM, Ing FF, de Moor M, Morrow WR, Mullins CE, Taubert KA, Zahn EM. Indications for cardiac catheterization and intervention in pediatric cardiac disease: A scientific statement from the American Heart Association. Circulation 2011;123:2607-2652.

2. Kobayashi D, Singh HR, Turner DR, Forbes TJ, Gowda ST. Transcatheter retrieval and repositioning of embolized stent from the right ventricle in an infant. Tex Heart Inst J 2012;39: 639-643.

3. Portugaller HR, Pabst E, Doerfler OC, Tauss J, Zangrando M, Pilger E, Klein GE. Crimping and repositioning of a maldeployed balloon-expandable arterial stent using a gooseneck snare. J Endovasc Ther 2005;12:247-251.

4. Slonim SM, Dake MD, Razavi MK, Kee ST, Samuels SL, Rhee JS, Semba CP. Management of misplaced or migrated endovascular stents. J Vasc Interv Radiol 1999;10:851-859.

5. Ghanem A, Tiemann K, Nickenig G. Gone with the flow: percutanous retrieval of a migrated wallstent trapped in the right ventricle. Eur Heart J 2009;30:717.

6. Narsupalli S, Ahmad Z, Veldtman G. Two-stent telescoping technique for securing an atrially embolized venous stent. J Invasive Cardiol 2010;22:E168-E170. 